\title{
Inhalt
}

Die beigefugten Zahlen beziehen sich auf die Seite

Einleitung . . . . . . . . . . . . . . . . . . . . 1

Tabellarische Übersicht der Perioden und Formationen . . . . 5

Erster Teil.

Pflanzenreich.

Systematische Übersicht . . . . . . . . . . . . . . . . . . . 10

I. Cryptogamae. Sporenpflanzen.

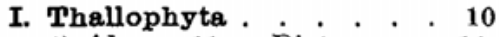

1) Algae 11. Diatomaceae 11. Siphoneae 11. Florideae 14. Characeae 15. Anhang: Receptaculitidae 15.

2) Fungi 16.

II. Bryophyta
III. Pteridophyta

1) Filicaceae 16.

2) Rhizocarpeac 18.

3) Calamarieae 19.

4) Sphenophylleae 22.

5) Lycopodiaceae 22. Lepidodendreae 22. Sigillarieae 24.

\section{Phanerogamae. Blütenpflanzen.}

I. Gymnospermae

1) Cycadaceae 26.

2) Cordaiteae 27 .

3) Coniferae 28.
25

II. Angiosperma

1) Monocotyledoneae 32 .

2) Dicotyledoneae 32 .

\section{Zweiter Teil.}

\section{Tierreich.}

Systematische Übersicht .

\section{Evertebrata. Wirbellose Tiere.}

I. Protozoa . . . . . . . 35

1) Rhizopoda 35 .

Foraminifera 35 .

Radiolaria 43.

II. Coelenterata.

1) Spongiae 45. Myxospongiae Ceraospongiae 46. Silicispongiae 47. Monactinellida 48. 35. Tetractinellida 48. Lithistidae 48. Hexactinellida 52. Calcispongiae 56. Sycones 56 . Pharetrones 57.

2) Cnidaria 57. Anthozoa 57 . Alcyonaria 61 . Tabulata 62. Zoantharia 64. Madreporaria
64. Tetracoralla 64. Hexacoralla 67. Hydrozoa 72. Hydroidea 73. Hydrocorallinae 73. Tabulariae 73. Campanulariae 74. Acalephae 77.

III. Echinodermata . . . . . 77

1) Crinoidea 77. Eucrinoidea 77. Cystoidea 84. Blastoidea 85 .

2) Asteroídea 87. Stelleridea 88. Ophiuroidea 88.

3) Echinoidea 89. Palechinoidea 91. Euechinoidea 92. Regulares Desor 92. Irregulares DESOR 94.

4) Holothurioidea 96 . 
IV. Vermes . . . . . . . . . . 96

V. Molluscoidea . . . . . . 97

1) Bryozoa 97. Cryptostomata 99. Cyclostomata 99. Cheilostomata 100.

2) Brachlopoda 100 . Inarticulata 103. Articulata 104.

VI. Mollusca . . . . . . . . 109

1) Lamellibranchiata 109. Asiphonida 112. Monomyaria 112. Heteromyaria114. Homomyaria 116. Siphonida 118. Integripalliata 118. Sinupalliata 124.

2) Scaphopoda 126.

3) Amphineura(Placophora)126.

4) Gastropoda127. Prosobranchia 129. Heteropoda 136. Opisthobranchia 136. Pulmonata 136.

5) Pteropoda 137. Thecosomata 137.

\section{Vertebrata.}

I. Pisces . . . . . . . 184 Selachii 186. Placodermi 188. Dipnoi 189. Ganoidei 190. Teleostei 192.

II. Amphibia . . . . . . 193 Stegocephala 194. Phyllospondyli 195. Lepospondyli 196. Temnospondyli 196. Stereospondyli 196. Urodela 198. Anura 198.

III. Reptilia . . . . . . . 199 Rhynchocephalia 200. Lepidosauria 200. Ichthyosauria 201. Sauropterygia 202. Theromorpha 202. Testudinata 203. Crocodilia 203. Parasuchia 204. Eusuchia 204. Pseudosuchia 204. Dinosauria 204. Sauro-
6) Cephalopoda 138. Tetrabranchiata 139. Nautiloidea 142. Ammonoidea 145. Dibranchiats 156. Belemnoidea 157. Sepioidea 160.

VII. Arthropoda $\quad . \quad . \quad . \quad . \quad .160$

1) Crustacea 160. Entomostraca 161. Cirripedia 161. Ostracoda 161. Phyllopoda 163. Trilobitae 163. Malacostraca 171. Leptostraca 171. Arthrostraca 172. Isopoda172. A mphipoda 172. Thoracostraca 172. Decapoda 172. Macrura 173. Brachyura 173. Merostomata 174. Gigantostraca 174. Xiphosura 175.

2) Myriapoda 176 .

3) Arachnoidea 176.

4) Insecta 177.

\section{Wirbeltiere.}

poda 205. Theropoda 205. Orthopoda205. Pterosauria 207.

IV. Aves . . . . . . . . 207 Saururae 208. Ratitae 209. Dinornithidae 209. Carinatae 210.

v. Mammalia . . . . . . 210 Monotremata 212. Marsupialia 212. Placentalia 213. Insectivora 213. Chiroptera 213. Carnivora 214. Creodontia 214. Fissipedia 214. Cetacea 215. Edentata 216. Rodentia 216. Ungulata 217. Amblypoda 217. Proboscidia 217. Perissodactyla 219. Artiodactyla 223. Prosimiae 226. Primates 227. 